\title{
CO AND THE MULTIPHASE ISM
}

\author{
CHRISTOPHER F. MCKEE \\ Departments of Physics and Astronomy \\ University of California \\ Berkeley CA 94720
}

\begin{abstract}
CO observations indicate that molecular clouds have a complex multiphase structure, and this is compared with the multiphase structure of the diffuse interstellar medium. The trace ionization within the molecular gas is governed primarily by UV photoionization. Magnetic fields contribute a significantly larger fraction of the pressure in molecular clouds than in the diffuse interstellar medium. Observations suggest that the total Alfvén Mach number, $m_{A}$ tot, of the turbulence in the diffuse ISM exceeds unity; Zeeman observations are consistent with $m_{A}$ tot $\lesssim 1$ in molecular clouds, but more data are needed to verify this. Most molecular clouds are selfgravitating, and they can be modeled as multi-pressure polytropes with thermal, magnetic, and wave pressure. The pressure and density within selfgravitating clouds is regulated by the pressure in the surrounding diffuse ISM.
\end{abstract}

\section{Introduction}

The molecular gas traced by $\mathrm{CO}$ is ubiquitous in the Galaxy. In order to understand how this gas fits into the interstellar medium (ISM) and is related to star formation, one must consider a wide range of scales, from the Galaxy as a whole down to small, dense cores that are undergoing gravitational collapse. Large scale phenomena, particularly spiral density waves, are crucial for the formation of molecular clouds (e.g., Elmegreen 1991), whereas the small scale phenomena determine the properties of individual young stellar objects (Shu, Adams, \& Lizano 1987). However, phenomena at intermediate scales determine which gas will form stars, the mass of the resulting stars (the initial mass function), and the star formation rate. It is also at these intermediate scales that $\mathrm{CO}$ observations provide the most 
information. These observations have shown that most of the $\mathrm{CO}$ is organized into giant molecular clouds (GMCs) that occupy a small fraction of the volume of the ISM (e.g., Scoville \& Sanders 1987). Furthermore, the molecular gas within GMCs is confined to clumps that occupy a small fraction of the volume of the GMC (Zuckerman \& Palmer 1974).

In this paper, I shall discuss several topics related to $\mathrm{CO}$ on intermediate scales: How are molecular clouds related to the multiphase ISM? In particular, what is the relative importance of the magnetic field in molecular gas compared to that in the diffuse ISM? How can molecular clouds and the clumps within them be modeled when several types of pressurethermal, magnetic, and turbulent-are present? Space precludes discussion of another topic, star formation in a clumpy GMC; the interested reader is referred to Bertoldi \& McKee (1996).

\section{GMCs and the Multiphase ISM}

The diffuse interstellar medium exhibits a complex structure that is generally described in terms of a multiphase medium (Field, Goldsmith, \& Habing 1969; McKee \& Ostriker 1977; McKee 1995). Cold H I clouds $\left(T \sim 10^{2} \mathrm{~K}\right.$-the $\left.\mathrm{CNM}\right)$ occupy a small fraction of the volume, whereas warm H I $\left(T \sim 10^{4} \mathrm{~K}\right)$, both neutral (WNM) and ionized (WIM), occupies a significant fraction of the volume. In the three-phase model of the ISM, hot gas $\left(T \sim 10^{6} \mathrm{~K}\right)$ fills somewhat over half the volume.

GMCs have a complex structure as well, although it is less well understood than that of the diffuse ISM. Much of the mass in GMCs is concentrated in clumps that are apparent in ${ }^{13} \mathrm{CO}$ and that occupy a few percent of the volume (e.g., Blitz \& Shu 1980; Bertoldi \& McKee 1992; Williams, deGeus, \& Blitz 1994). The nature of the interclump medium is uncertain: both cold H I (Blitz 1991) and warm H I (Falgarone \& Puget 1986; McKee 1989) have been suggested. It is thus quite possible that molecular clouds are themselves three phase media, composed of $\mathrm{H}_{2}, \mathrm{CNM}$, and WNM (McKee 1995). Just as there is a substantial range in density observed in the diffuse ISM (Jenkins et al 1983), so too does the density of molecular gas extend over a considerable range. In part, this is due to the effects of self gravity, but very dense molecular gas is also observed on small scales where the effects of self-gravity are negligible (Falgarone et al 1991).

To continue the comparison between the diffuse, atomic ISM and the dense, molecular ISM, consider the energy sources. In the diffuse gas, UV starlight provides the energy for the photoelectric heating that is dominant for both the cold and warm phases (see Wolfire et al 1995 for a recent discussion). The same is true for the outer parts of molecular clouds; in the inner parts, however, which are shielded from the UV radiation, cosmic 
ray heating dominates. The primary energy source for nonthermal motions in the diffuse ISM is supernovae; in molecular clouds, it is outflows from young stellar objects (Norman \& Silk 1980).

Next, consider the source of the ionization. In the diffuse ISM, cosmic rays and X-rays ionize the $\mathrm{H} \mathrm{I}$, whereas EUV radiation, primarily from hot stars, maintains the ionization of the H II. In the molecular gas, the ionization is very weak; FUV radiation from stars ionizes the outer parts of molecular clouds, whereas cosmic rays ionize the cores. Most molecular gas in the ISM is in the outer, photoionized regions (McKee 1989).

\subsection{MAGNETIC FIELDS IN THE DIFFUSE ISM AND IN GMCS}

Finally, we consider magnetic fields. For the diffuse ISM, Boulares \& Cox (1990) have determined that the total pressure in the midplane of the Galaxy in the solar vicinity is $P_{\text {tot }} / k \simeq 28000 \mathrm{~K} \mathrm{~cm}^{-3}$. Of this, they estimated that cosmic rays contribute about $7000 \mathrm{~K} \mathrm{~cm}^{-3}$ and thermal plus turbulent pressures about $11000 \mathrm{~K} \mathrm{~cm}^{-3}$. As a result, they concluded that the remaining pressure of $10000 \mathrm{~K} \mathrm{~cm}^{-3}$ is due to the magnetic field, corresponding to $B=5.8 \mu \mathrm{G}$. (They also considered a model with a higher total pressure and a yet stronger field.) Subsequent analysis has failed to confirm such large magnetic pressures, however. In a recent review, Heiles (1996) concludes that the magnetic field in the solar vicinity consists of a uniform component $B_{u} \simeq 2.2 \mu \mathrm{G}$ and a random component $B_{r} \simeq 3.6$ $\mu \mathrm{G}$, corresponding to a total field $B_{\text {tot }}=\left(B_{u}^{2}+B_{r}^{2}\right)^{1 / 2} \simeq 4.2 \mu \mathrm{G}$. Pulsar observations suggest that the correlation length of the random component of the field is in the range 10-100 pc in the Galactic plane (Ohno \& Shibata 1993). Little is known about the structure of $B_{r}$ out of the Galactic plane; Boulares \& Cox suggested that the vertical component of the field $B_{z}$ (which has a random sign) increases systematically with height so as to account for observations of the Galactic synchrotron radiation.

Because the field has a random component, it exerts a smaller force than a uniform field. The force due to a uniform component of the field $B_{u}$ is simply $-\nabla B_{u}^{2} / 8 \pi$. However, the large-scale force exerted in the $z$ direction, say, by the random component $B_{r}$ is $-\partial / \partial z\left(B_{r \text { eff }}^{2} / 8 \pi\right)$, where

$$
B_{r \text { eff }}^{2}=\left\langle B_{r}^{2}-2 B_{r z}^{2}\right\rangle
$$

(Parker 1969; Boulares \& Cox 1990). In deriving this result, it has been assumed that the force is averaged over a volume large enough that the averages $\left\langle B_{r x} B_{r z}\right\rangle$ and $\left\langle B_{r y} B_{r z}\right\rangle$ both vanish. The effective magnetic pressure on large scales is then

$$
P_{\text {Beff }}=\frac{1}{8 \pi}\left(B_{u}^{2}+\frac{1}{3} B_{r}^{2}\right)
$$


if $\vec{B}_{r}$ is isotropic (which is reasonable near the Galactic plane at least), so that $B_{r \text { eff }}^{2}=B_{r}^{2} / 3$. For the field strengths found by Heiles (1996), this means that the large-scale effective field pressure is only $P_{B}$ eff $/ k=2640$ $\mathrm{K} \mathrm{cm}^{-3}$, less than $10 \%$ of the total pressure. The full magnetic pressure $P_{B \text { tot }}=B_{\text {tot }}^{2} / 8 \pi \simeq 5000 \mathrm{~K} \mathrm{~cm}^{-3}$ is effective only on scales smaller than the correlation length of the field; it is about $18 \%$ of the total pressure. In either case, the magnetic pressure is substantially smaller than that inferred by Boulares \& Cox.

If the magnetic field does not support the ISM, what does? Most likely, turbulent motions in the interstellar gas provide the necessary support (McKee 1990; Lockman \& Gehman 1991). With a thermal pressure of $3600 \mathrm{~K}$ $\mathrm{cm}^{-3}$ (Jura 1975), the turbulent pressure required is $15000 \mathrm{~K} \mathrm{~cm}^{-3}$. The Alfvén Mach number $m_{A}$ defined in terms of $B_{u}$ and the 1-D nonthermal velocity dispersion $\sigma_{\mathrm{nt}}$,

$$
m_{A}^{2}=\frac{3 \sigma_{\mathrm{nt}}^{2}}{v_{A}^{2}}=\frac{3}{2}\left(\frac{\rho \sigma^{2}}{B_{u}^{2} / 8 \pi}\right),
$$

is then quite large, $m_{A} \simeq 4$. It is often stated that the Alfvén Mach number should be less than or of order unity since the dissipation becomes very large for super-Alfvénic motions. However, it is important to distinguish between $m_{A}$, which is defined in terms of the uniform field $B_{u}$, and $m_{A}$ tot $\propto 1 / B_{\text {tot }}$, which is defined in terms of the total field strength $B_{\text {tot }}$. Once a wave steepens to the point that its wavelength is less than a correlation length in the field, it will feel the full magnetic pressure $B_{\text {tot }}^{2} / 8 \pi$. Shocks will not form until $m_{A}$ tot exceeds unity; for the ISM as a whole, we infer $m_{A}$ tot $\simeq 2$. Although $m_{A}$ tot exceeds unity, it is not clear that shocks will form, since a significant fraction of the turbulent pressure is in clouds that are moving sub-Alfvénically through the intercloud medium.

In GMCs, the total pressure is much greater than in the diffuse ISM (Scoville \& Sanders 1987; Blitz 1991). As a result, the thermal pressure, which appears to be comparable to that in the diffuse medium (Falgarone \& Puget 1986), and the cosmic ray pressure represent a smaller fraction of the total pressure in GMCs than in the diffuse ISM. Furthermore, the cosmic ray pressure is approximately uniform, so that cosmic rays exert almost no force. As a result, the total pressure in GMCs is dominated by dynamical pressure and magnetic pressure,

$$
P \simeq \rho \sigma_{\mathrm{nt}}^{2}+\frac{B_{u}^{2}}{8 \pi}+\frac{1}{3} \frac{B_{r}^{2}}{8 \pi} .
$$

Here we have assumed that $B_{r}$ is isotropic; note that $B_{r}$ may include a time dependent part $\delta B$ associated with wave motions as well as a static part. 
Only the uniform component of the field $B_{u}$ is accessible to observation through the Zeeman effect (Zweibel \& McKee 1995).

Analysis of the observations of a number of molecular clouds by Myers \& Goodman (1988) showed that typically $m_{A} \sim 1$; the uncertainties inherent in this result were discussed by Heiles et al (1993). More recently, however, Crutcher et al (1993) searched for the Zeeman effect in $\mathrm{OH}$ in a number of molecular clouds and for the most part found only upper limits. Their data show that the harmonic mean $m_{A}$ in 10 clouds with $1 \sigma$ errors less than 4 $\mu \mathrm{G}$ is 1.5 , so that the mean $m_{A}$ is somewhat higher, perhaps about 2 . If the total field is about twice the uniform field, as it is in the diffuse ISM, then the data of Crutcher et al are consistent with $m_{A}$ tot $\lesssim 1$, so that the observed motions are sub-Alfvénic with respect to $B_{\text {tot }}$. In this case, the magnetic pressure must be a substantial fraction of the total pressure in molecular clouds: equation (4) implies that in GMCs

$$
\frac{P_{B \text { tot }}}{P} \gtrsim \frac{1}{1+\frac{2}{3} m_{A \text { tot }}^{2}}
$$

so that the total magnetic pressure would be at least $60 \%$ of the total pressure. It would be very valuable to make more sensitive observations of the magnetic field in molecular clouds in order to verify this.

We conclude that the total magnetic pressure in the diffuse ISM is less than about $20 \%$ of the total pressure, whereas in molecular clouds it is typically of order $60 \%$. The increased role of magnetic pressure in molecular clouds is due to two factors: (1) the total pressure is much larger, so the thermal and cosmic ray pressure constitute a smaller fraction; and (2) the Alfvén Mach number associated with the turbulent motions in the clouds appears to be smaller than in the diffuse ISM. This latter effect can be understood in terms of the equipartition arguments developed by Zweibel $\&$ McKee (1995; see also Zweibel 1995). They showed that in the absence of damping the kinetic energy in the waves, which is $(3 / 2) \rho \sigma_{\mathrm{nt}}^{2}$ after averaging over direction, must equal the potential energy associated with the restoring forces that drive the waves. For transverse waves, which suffer less damping than longitudinal waves, the potential energy consists of the fluctuating field energy, $\delta B^{2} / 8 \pi$, and the tidal gravitational energy, which they wrote as $\delta_{2} w_{G}$ (tidal). Equipartition then implies that $m_{A}$ tot is given by

$$
m_{A \text { tot }}^{2} \simeq \frac{\delta B^{2}}{B_{\text {tot }}^{2}}+\frac{\delta_{2} w_{G}(\text { tidal })}{B_{\text {tot }}^{2} / 8 \pi} .
$$

In the diffuse ISM, stars, not gas, dominate the gravitational field. As a result, the tidal term can be larger in the diffuse ISM than in molecular clouds, enabling $m_{A}$ tot to be larger there as well. 


\section{Multi-Pressure Polytrope Models of Molecular Clouds}

Most molecular gas in the Galaxy is self-gravitating (Larson 1981), and as a result its pressure is significantly greater than that of the diffuse ISM. Just as in the case of stars, it is therefore convenient to make polytropic models of molecular clouds and the clumps within them by assuming that the pressure scales as some power of the density, $P \propto \rho^{\gamma_{\mathrm{p}}}$ (Dickman \& Clemens 1983; Chieze 1987; Maloney 1988; Elmegreen 1989). Previous polytropic models of molecular clouds suffered from the problem that it is difficult to determine the appropriate value of the polytropic index $\gamma_{\mathrm{p}}$. The simplest approach is to assume that the cloud is isothermal $\left(\gamma_{p}=1\right)$; in that case, one finds that the maximum mass that the cloud can have is the BonnorEbert mass, $M_{\mathrm{BE}}=1.18 \sigma^{4} /\left(G^{3} P_{0}\right)^{1 / 2}$ (Bonnor 1956; Ebert 1955). Such a model makes no allowance for the effect of magnetic fields or turbulent motions, however, and is in conflict with the observation that the velocity dispersion in a cloud increases with scale (Larson 1981). In response to this problem, Maloney (1988) suggested that $\gamma_{\mathrm{p}}$ should be less than unity, so that the clouds would be hotter on the outside than in the center. The difficulty with this type of model is that the mean pressure in the clouds is then not much greater than at the surface, which again is in conflict with observation.

To overcome these problems, Holliman \& McKee (1996; hereafter HM) have introduced multi-pressure polytropes that treat all the important sources of pressure in molecular clouds: thermal motions $\left(P_{\mathrm{th}}\right)$, static magnetic fields $\left(P_{B}\right)$, and fluctuating fields and velocities $\left(P_{\mathrm{w}}\right)$. This last pressure is modeled as being due to a superposition of Alfvén waves (Arons \& Max 1975; McKee \& Zweibel 1995). The main limitation of this polytropic model is the assumption of spherical symmetry. However, as shown by Mouschovias \& Spitzer (1976), spherical models can be used to determine the stability of self-gravitating, magnetized clouds with reasonable accuracy. HM show that in the absence of ambipolar diffusion, static fields can be represented by $\gamma_{\mathrm{p}}=4 / 3$; ambipolar diffusion leads to $\gamma_{\mathrm{p}}<4 / 3$. The pressure due to the Alfvén waves, $P_{w}=(3 / 2) \rho \sigma_{\mathrm{nt}}^{2}$, is isotropic, and it includes both the dynamical pressure and the pressure due to the fluctuating magnetic field (McKee \& Zweibel 1995). The polytropic index for the waves is $1 / 2$. At any point, the pressure in the cloud is

$$
P(r)=\Sigma K_{i} \rho(r)^{\gamma_{p, i}},
$$

where the sum extends over the three components of the pressure.

The stability of the polytropes is governed by the adiabatic index $\gamma$, which describes the response of the gas to a compression, as well as by the polytropic index $\gamma_{p}$. For an isothermal gas, $\gamma=1$; for the static field, 
$\gamma=4 / 3$, which ensures that the relative contribution of the field to the support of the cloud is independent of compression; and, for Alfvén waves, $\gamma=3 / 2$ (McKee \& Zweibel 1995). HM show that a central result from the study of single-pressure polytropes (Chieze 1987, Elmegreen 1989) remains valid for multi-pressure polytropes: the pressure and density within a cloud are regulated by the pressure at its surface. Only magnetically subcritical clouds, which remain stable for arbitrarily high densities, can escape this conclusion. Observed clouds, however, derive a significant amount of support from thermal pressure and waves, and are therefore magnetically supercritical (McKee 1989). Thermal pressure and wave pressure each have $\gamma<4 / 3$ and $\gamma_{p}<1.2$, and HM show that this places an upper bound on the ratio of central to surface pressures for stable molecular clouds. Molecular clouds are thus the opposite of stars, which can exist in a zero pressure environment.

Observations show that GMCs have relatively large pressure ratios. For self-gravitating clouds, the mean pressure is $\bar{P} / k \simeq 5000 \bar{A}_{V}^{2} \mathrm{~K} \mathrm{~cm}^{-3}$, where $\bar{A}_{V}$ is the mean extinction through the cloud (McKee 1989). Solomon et al (1987) inferred $\bar{A}_{V} \simeq 7.5$ in the sample of GMCs they observed. This corresponds to a mean pressure of about $3 \times 10^{5} \mathrm{~K} \mathrm{~cm}^{-3}$, ten times the local interstellar pressure. By contrast, an isothermal sphere has a mean pressure that is at most 2.5 times the surface value. HM show that when the shielding layer of atomic gas around the molecular cloud is taken into account (Elmegreen 1989), multi-pressure polytropes can account for the large observed pressure ratio.

Multi-pressure polytropes have also been used to model low-mass cores. These models improve upon the phenomenological "TNT" models of Myers \& Fuller (1992) by including the effects of the static magnetic field and by integrating the equation of hydrostatic equilibrium. HM find models that span a factor of about 100 in density from the center of the core to the surface, considerably greater than the factor 14 possible for stable isothermal clouds that are supported by gas pressure alone. The density profile in the envelope is somewhat steeper than $r^{-2}$, the profile for a singular isothermal sphere. The results for $\sigma_{\mathrm{nt}}(r)$ are consistent with the observations of Fuller \& Myers (1992) except at the center, where the omission of wave damping in the model leads to a higher value of $\sigma_{\mathrm{nt}}$ than observed. Application of the model to high-mass cores gives a flatter $\sigma_{\text {nt }}(r)$ relation than for low-mass cores, as observed by Caselli \& Myers (1995).

\section{Acknowledgements}

My research is supported by NSF grant AST 92-21289, and, for research on star formation, by a NASA grant to the Center for Star Formation Studies. 


\section{References}

Arons, J., \& Max, C. E. 1975, ApJ, 196, L77.

Bertoldi, F., and McKee, C.F. 1992, ApJ, 395, 140.

Bertoldi, F., and McKee, C.F. 1996, to be submitted to ApJ.

Blitz, L. 1991, in The Physics of Star Formation and Early Stellar Evolution, eds.

C.J. Lada and N.D. Kylafis (Dordrecht: Kluwer), p.3.

Blitz, L., \& Shu, F. H. 1980, ApJ, 238, 148.

Bonnor, W. B. 1956, MNRAS, 116, 351.

Boulares, A., \& Cox, D. P. 1990, ApJ, 365, 544.

Caselli, P., \& Myers, P. C. 1995, ApJ, 446, 665.

Chieze, J. P. 1987, A\&A, 171, 225.

Crutcher, R. M. 1993, ApJ, 407, 175.

Dickman, R. L., \& Clemens, D. P. 1983, ApJ, 271, 143.

Ebert, R. 1955, Z. Astrophys., 37, 222.

Elmegreen, B. G. 1989, ApJ, 338, 178.

Elmegreen, B. G. 1991, ApJ, 378, 139.

Falgarone, E., \& Puget, J. L. 1986, A\&A, 162, 235.

Falgarone, E., Phillips, T. G., \& Walker, C. K. 1991, ApJ, 378, 186.

Field, G. B., Goldsmith, D. W., \& Habing, H. J. 1969, ApJ, 155, L149.

Fuller, G. A., \& Myers, P. C. 1992, ApJ, 384, 523.

Heiles, C. 1996, in Polarimetry of the Interstellar Medium, ed. W. G. Roberge \& D. C. B. Whittet (San Francisco: ASP), in press.

Heiles, C., Goodman, A., McKee, C. F., \& Zweibel, E. 1993, in Protostars and Planets III, eds. E. Levy and J. Lunine (Tucson: University of Arizona Press), p.279.

Holliman, J. H., \& McKee, C. F. 1996, ApJ, to be submitted (HM).

Jenkins, E. B., Jura, M., \& Loewenstein, M. 1983, ApJ, 270, 88.

Jura, M. 1975, ApJ, 197, 581.

Larson, R. B. 1981, MNRAS, 194, 809.

Lockman, F. J., \& Gehman, C. S. 1991, ApJ, 382, 182.

Maloney, P. 1988, ApJ, 334, 761.

McKee, C. F. 1989, ApJ, 345, 782 .

McKee, C. F. 1990, in The Evolution of the Interstellar Medium, ed. L. Blitz (San Francisco: ASP), p. 3.

McKee, C. F. 1995, in The Physics of the Interstellar Medium and Intergalactic Medium, ed. A. Ferrara, C. F. McKee, C. Heiles, \& P. Shapiro (San Francisco: ASP), p. 292.

McKee, C. F., \& Ostriker, J. P. 1977, ApJ, 218, 148.

McKee, C. F., \& Zweibel, E. G. 1995, ApJ, 440, 686.

Mouschovias, T., \& Spitzer, L. 1976, ApJ, 210, 326.

Myers, P. C., \& Fuller, G. A. 1992, ApJ, 392, 631.

Myers, P. C., \& Goodman, A. A. 1988, ApJ, 326, L27.

Norman, C. A., \& Silk, J., 1980, ApJ, 238, 158.

Ohno, H., \& Shibata, S. 1993, MNRAS, 262, 953.

Parker, E. N. 1969, Space Sci. Rev., 9, 651.

Scoville, N. Z., \& Sanders, D. B. 1987, in Interstellar Processes, eds. D. Hollenbach and H. Thronson (Dordrecht: Reidel), p.21.

Shu, F. H.. Adams, F. C., \& Lizano, S. 1987, ARA\&A, 25, 23.

Solomon, P. M., Rivolo, A. R., Barrett, J. W., \& Yahil, A. 1987, ApJ, 319, 730.

Williams, J. P., deGeus, E. J., \& Blitz, L. 1995, ApJ, 428, 693.

Wolfire, M. G., Hollenbach, D. J., McKee, C. F., Tielens, A. G. G. M., \& Bakes, E. L. O. 1995, ApJ, 443, 152.

Zuckerman, B., \& Palmer, P. 1974, ARA\&A, 12, 279.

Zweibel, E. G. 1995, in The Physics of the Interstellar Medium and Intergalactic Medium, ed. A. Ferrara, C. F. McKee, C. Heiles, \& P. Shapiro (San Francisco: ASP), p. 524.

Zweibel, E. G., \& McKee, C. F. 1995, ApJ, 439, 779. 